\title{
Expression of microRNA-106b and its clinical significance in cutaneous melanoma
}

\author{
N. Lin, Y. Zhou, X. Lian and Y. Tu \\ Department of Dermatology, Union Hospital, Tongji Medical College, \\ Huazhong University of Science and Technology, Wuhan, Hubei, China \\ Corresponding author: N. Lin \\ E-mail: xh_linnengxing@126.com \\ Genet. Mol. Res. 14 (4): 16379-16385 (2015) \\ Received August 8, 2015 \\ Accepted October 7, 2015 \\ Published December 9, 2015 \\ DOI http://dx.doi.org/10.4238/2015.December.9.6
}

ABSTRACT. MicroRNA-106b (miR-106b) is overexpressed in various types of cancers and is associated with the regulation of carcinogenic processes. However, its clinical significance in cutaneous melanoma has not been reported. qRT-PCR was performed to examine the expression of miR-106b in 15 cases of dysplastic nevi, 17 cases of melanoma metastases, and 97 cases of primary cutaneous melanoma tissue samples. Survival rate was determined with Kaplan-Meier and statistically analyzed with the log-rank method between groups. Survival data were evaluated through multivariate Cox regression analysis. Significant differences in miR-106b expression were shown between dysplastic nevi and primary cutaneous melanomas $(P<0.01)$, between primary melanomas and metastatic cutaneous melanomas $(P<0.01)$, and between primary cutaneous melanomas and metastatic cutaneous melanomas $(P<0.001)$. We found that high miR106b expression was correlated with Breslow thickness $(P=0.002)$, tumor ulceration $(P=0.002)$, and advanced clinical stage $(P<0.001)$. The patients with high miR-106b expression showed shorter 5-year overall survival than those with low miR-106b expression ( $P=0.02$; log-rank test). Multivariate regression analysis showed that the status of miR-106b expression was an independent prognostic factor for overall survival $(\mathrm{HR}=2.09,95 \% \mathrm{Cl}$ : 1.11-10.26, $P=0.02$ ). This study showed that miR-106b may contribute 
to the progression of cutaneous melanoma and its up-regulation may be independently associated with poor prognosis of cutaneous melanoma. This suggests that miR-106b might serve as a promising biological marker for further risk stratification in the management of cutaneous melanoma.

Key words: MicroRNA-106b; Cutaneous melanoma; Prognosis; Biomarker

\section{INTRODUCTION}

Cutaneous melanoma is a common form of cutaneous malignancy arising from the pigment cells of the skin (Geller et al., 2002; Merrill, 2011). Aggressive invasion, early metastasis, and resistance to chemotherapy or radiotherapy characterize malignant melanoma, and this has caused an increase in its incidence and associated mortality worldwide (Terando et al., 2003). Therefore, a better understanding of the molecular mechanisms that mediate malignant melanoma tumorigenesis and progression is essential to the eventual design of novel therapeutic agents and the identification of prognostic markers for cutaneous melanoma.

MicroRNAs (miRNAs) are a set of small non-coding RNA molecules that regulate the expression of target genes (Lee et al., 1993). Specifically, partial complementary pairing between the 3'-untranslated region (3'UTR) of the messenger RNA (mRNA) and the 5' seed region of a miRNA is essential for the post-transcriptional modulation of the target gene expression (Karginov et al., 2007). Moreover, miRNA appear to play a role in carcinogenesis by modulating the expression of tumor suppressor genes and oncogenes to generate a complex combinatorial network (Cui et al., 2006; Bhatti et al., 2009; Wang et al., 2011).

MicroRNA-106b (miR-106b) is transcribed from the miR-106b-25 cluster located on chromosome (Hudson et al., 2013). This miRNA cluster is reported to be overexpressed in hepatocellular carcinoma (HCC) clinical samples (Li et al., 2009). Moreover, a microarray study demonstrated that miR-106b was up-regulated in colon cancer with lymph node metastasis (Wang et al., 2010). miR-106b can directly bind to p21 mRNA and regulate cell cycle progression (Kan et al., 2009). It can also regulate the E-cadherin distribution in embryonic lung epithelial branching morphogenesis through regulating the expression of MAPK14 and STAT3 (Carraro et al., 2009). The role of miR-106b has also been investigated in melanoma, when Prasad and Katiyar (2014) found that miR-106b played a crucial role in melanoma growth. However, its clinical significance in cutaneous melanoma has not been reported.

\section{MATERIAL AND METHODS}

\section{Study population and tissue samples}

This study was approved by the local Ethical Committee, and informed written consent was obtained from all patients. A total of 15 cases of dysplastic nevi, 17 cases of melanoma metastases, and 97 cases of primary cutaneous melanoma tissue samples were collected directly from surgery after removal of the necessary amount of tissue for routine pathology examination in the Department of Pathology, Union Hospital, Tongji Medical College, Huazhong University of Science and Technology between May 2005 and March 2013. The histological diagnosis, Breslow thickness, and Clark level were re-examined for 1-5 of the original sections of the primary tumor by the same pathologist who was unaware of the clinical data. The tumors were frozen at $-80^{\circ} \mathrm{C}$ in $a$ 
guanidinium thiocyanate solution and RNA was extracted from the samples using a standard Trizol RNA extraction method (Invitrogen, Life Technologies, Carlsbad, CA, USA). The characteristics of the patients are shown in Table 1.

\section{Quantitative reverse transcriptase PCR (qRT-PCR)}

The expression of miR-106b was determined by qRT-PCR. Briefly, total RNA was extracted from the tissues using TRIzol reagent (Invitrogen) according to the manufacturer's protocol. miRNA expression levels were then quantitated using the TaqMan miRNA real-time RT-PCR kit (Applied Biosystems, USA) according to the manufacturer protocol. Data were analyzed using 7500 software v.2.0.1 (Applied Biosystems), with the automatic Ct setting for adapting the baseline and threshold for $\mathrm{Ct}$ determination. The universal small nuclear RNA U6 (RNU6B) was used as an endogenous control for miRNAs. Each sample was examined in triplicate, and the quantities of PCR products produced were normalized o RNU6B.

\section{Statistical analysis}

All statistical analyses were performed using the SPSS 18.0 software (SPSS, Chicago, IL). Associations between miR-106b expression and various clinicopathological characteristics were analyzed using the $\chi^{2}$ test. Survival times were counted from the date of presentation to the date of death or last follow-up time. Survival rate was determined with Kaplan-Meier and statistically analyzed with the log-rank method between groups. Survival data were evaluated through multivariate Cox regression analysis. $P$ values of $<0.05$ were considered statistically significant.

\section{RESULTS}

\section{The expression level of miR-106b in cutaneous melanoma}

qRT-PCR was performed to examine the expression of miR-106b in 15 cases of dysplastic nevi, 17 cases of melanoma metastases, and 97 cases of primary cutaneous melanoma tissue samples. We found that there were significant differences in the pattern of relative miR-106b expression (Figure 1). Significant differences for miR-106b expression were shown between dysplastic nevi and primary cutaneous melanomas $(P<0.01)$, between primary melanomas and metastatic cutaneous melanomas $(P<0.01)$, and between primary cutaneous melanomas and metastatic cutaneous melanomas $(P<0.001)$.

\section{Correlation between miR-106b expression and clinicopathological variables of patients with cutaneous melanoma}

To investigate the correlation between miR-106b expression and clinicopathological variables of the patients, the expression of miR-106b in 97 primary cutaneous melanomas was examined. In this study, patients with values less than the median expression level in tumor tissues were assigned to the low expression group $(\mathrm{N}=47)$, whereas those with values more than the median expression level were assigned to the high expression group $(N=50)$. As shown in Table 1 , high miR-106b expression was correlated with Breslow thickness $(P=0.002)$, tumor ulceration $(P=0.002)$, and advanced clinical stage $(P<0.001)$. However, high miR-106b expression was not 
associated with other clinicopathological factors including age $(P=0.527)$, gender $(P=0.541)$, histologic type $(P=0.529)$, and tumor site $(P=0.418)$.

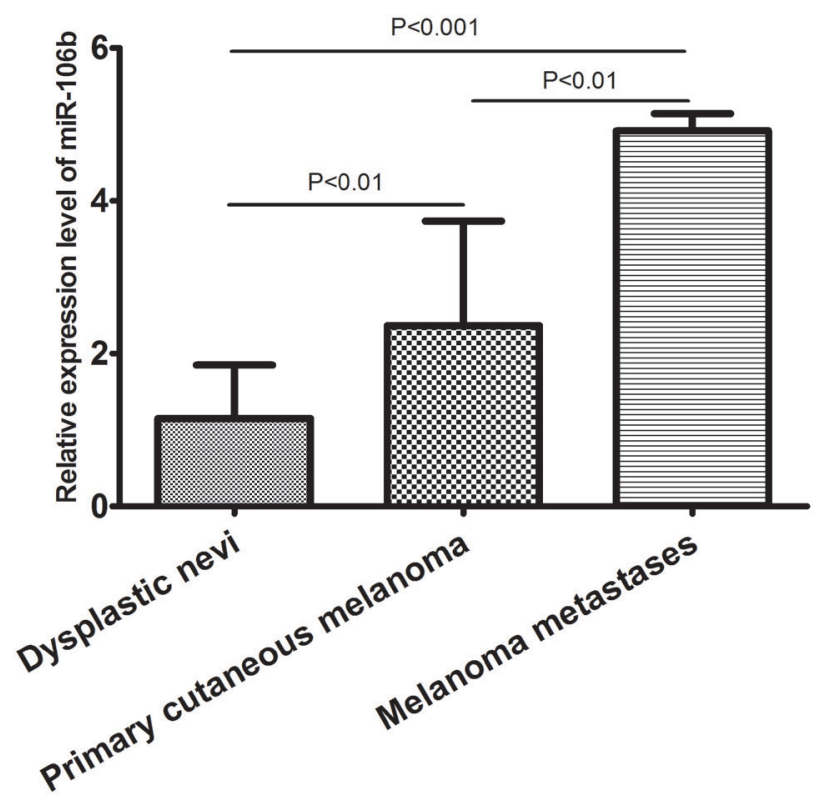

Figure 1. Expression level of miR-106b in 15 cases of dysplastic nevi, 17 cases of melanoma metastases, and 97 cases of primary cutaneous melanoma tissue samples.

Table 1. miR-106b expression level and clinicopathological factors in 97 patients with primary cutaneous melanomas.

\begin{tabular}{|c|c|c|c|c|}
\hline \multirow[t]{2}{*}{ Variables } & \multirow[b]{2}{*}{ Case $(\mathrm{N})$} & \multicolumn{2}{|c|}{ miR-106b expression level } & \multirow[t]{2}{*}{$P$ value } \\
\hline & & High $(N=50)$ & Low $(N=47)$ & \\
\hline \multicolumn{5}{|l|}{ Age } \\
\hline$\leq 55$ & 32 & 18 & 14 & 0.527 \\
\hline$>55$ & 65 & 32 & 33 & \\
\hline \multicolumn{5}{|l|}{ Gender } \\
\hline Male & 54 & 26 & 28 & 0.541 \\
\hline Female & 43 & 24 & 19 & \\
\hline \multicolumn{5}{|l|}{ Thickness (mm) } \\
\hline$\leq 2.0$ & 34 & 10 & 24 & 0.002 \\
\hline$>2.0$ & 63 & 40 & 23 & \\
\hline \multicolumn{5}{|l|}{ Ulceration } \\
\hline Absent & 30 & 8 & 22 & 0.002 \\
\hline Present & 67 & 42 & 25 & \\
\hline \multicolumn{5}{|l|}{ Histologic type } \\
\hline SSM & 44 & 23 & 21 & 0.529 \\
\hline LMM & 53 & 27 & 26 & \\
\hline \multicolumn{5}{|l|}{ Site } \\
\hline Sun exposed & 46 & 26 & 20 & 0.418 \\
\hline Sun protected & 51 & 24 & 27 & \\
\hline \multicolumn{5}{|l|}{ Stage } \\
\hline I/II & 40 & 9 & 31 & $<0.001$ \\
\hline III/IV & 57 & 41 & 16 & \\
\hline
\end{tabular}

LMM = lentigo malignant melanoma; SSM = superficial spreading melanoma. 


\section{Prognostic value of miR-106b in cutaneous melanomas}

To assess whether the expression of miR-106b was a tumor prognostic biomarker, the overall survival was investigated with respect to expression levels of miR-106b in primary cutaneous melanoma. Ninety-seven patients included in the study during the follow-up period and the survival curves plotted by Kaplan-Meier method were shown. As shown in Figure 2, the patients with high miR-106b expression showed shorter 5-year overall survival than those with low miR$106 \mathrm{~b}$ expression ( $P=0.02$; log-rank test). Multivariate regression analysis of the clinicopathological factors related to patient prognosis showed that the status of miR-106b expression was an independent prognostic factor for overall survival (Table 2; $\mathrm{HR}=2.09,95 \% \mathrm{Cl}$ : 1.11-10.26, $\mathrm{P}=$ 0.02). Thus, high miR-106b expression was correlated with the poorer overall survival of patients with cutaneous melanoma.

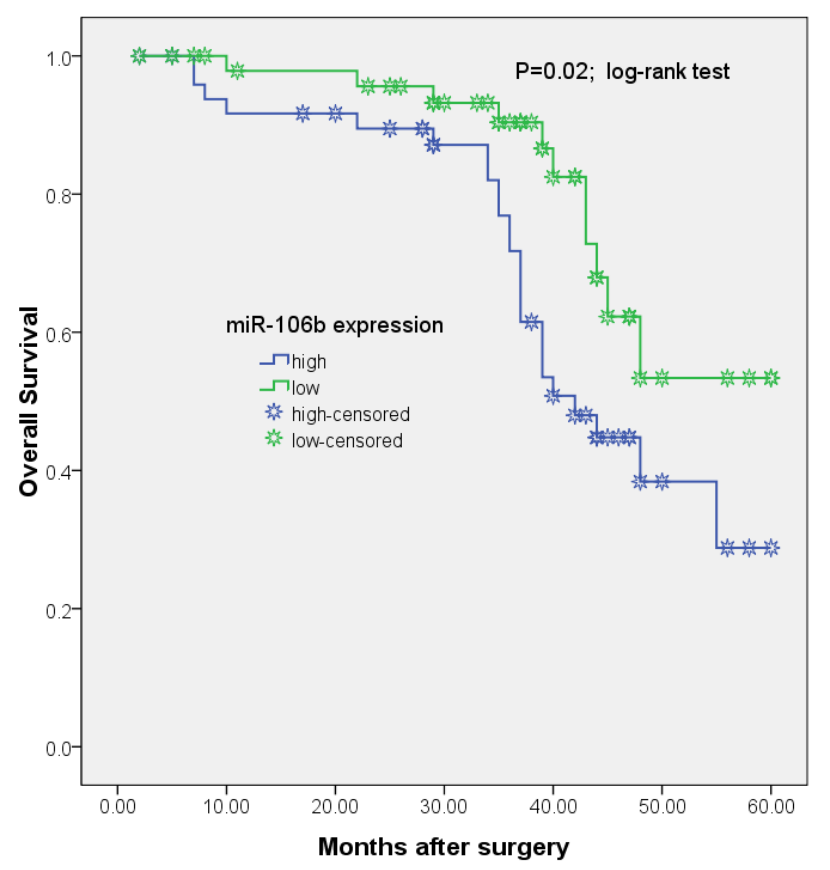

Figure 2. Kaplan-Meier survival curve of overall survival according to miR-106b expression level.

Table 2. Multivariate analyses of prognostic parameters in 97 patients with primary cutaneous melanomas by Cox regression analysis.

\begin{tabular}{lccc}
\hline Variable & Hazard ratio & $95 \%$ confidence interval & $P$ value \\
\hline Age & 1.22 & $0.26-1.78$ & 0.35 \\
Gender & 0.87 & $0.17-2.13$ & 0.72 \\
Thickness & 2.33 & $1.27-9.22$ & 0.01 \\
Ulceration & 1.98 & $0.88-6.92$ & 0.06 \\
Histologic type & 1.25 & $0.65-2.37$ & 0.48 \\
Site & 0.73 & $0.36-1.66$ & 0.48 \\
Stage & 3.94 & $1.84-14.89$ & 0.003 \\
miR-106b expression & 2.09 & $1.11-10.26$ & 0.02 \\
\hline
\end{tabular}




\section{DISCUSSION}

Cutaneous melanoma is the most severe form of skin cancer, and its incidence is on the rise. Early-stage melanoma can be cured by surgical resection, but late-stage melanoma is difficult to treat with current therapies. To improve treatment for melanoma patients, large efforts have been put into the elucidation of the cellular and molecular mechanisms underlying this disease (Luo et al., 2013).

Researchers are attempting to exploit and identify miRNAs that may serve as either diagnostic or prognostic markers, or therapeutic targets in many different tumor types (NanaSinkam and Croce, 2011). Although the functions of some miRNAs in a variety of human cancers have been identified, limited data are available about the changes in miRNA expression levels and their roles in cutaneous melanoma.

MiR-106b is overexpressed in various types of cancers and is associated with the regulation of carcinogenic processes. Wang et al. (year) found that miR-106b, at least in part, played a role in acquired malignant progression in pediatric brainstem glioma (BSG) compared with the adult group. Their study provided the evidence that miR-106b might serve as a causative agent in the malignant progression of pediatric BSG, thereby serving as a novel target for constraining the rapid and fatal course of pediatric BSG (Wang et al., 2012). Additionally, the study by Yau et al. (2013) demonstrated that miR-106b was overexpressed in HCC tissues as compared with adjacent non-tumor tissue $(\mathrm{P}=$ $0.0005)$, and overexpression of miR-106b was significantly correlated with a higher tumor grade $(P=$ 0.018). Further functional studies demonstrated that miR-106b could promote cell migration and stress fiber formation by overexpressing the RhoGTPases, RhoA and RhoC. In vivo functional studies also showed that overexpression of miR-106b promoted HCC metastasis (Yau et al., 2013). Moreover, Slaby et al. (2010) found that miRNA-106b was significantly overexpressed in renal cell carcinoma (RCC) tissues $(P<0.0001)$. In this case, miRNA-106b expression levels may act as a potential predictive marker of early metastasis after nephrectomy in RCC patients (long-rank $P=0.032$ ) (Slaby et al., 2010).

In addition to the role of miR-106b in the above cancers, this miRNA has also been investigated in melanoma. Previously, Prasad and Katiyar (2014) have observed the overexpression of miR-106b in various melanoma cell lines (A375, Hs294t, SK-Mel28, SK-Mel 119, Mel 1241, Mel 1011 and Mel 928) as compared to its expression in normal human epidermal melanocytes (NHEM). The overexpression of miR-106b in melanoma cells (A375, Hs294t) was associated with greater cell proliferation capacity as compared to NHEM. Treatment of A375 and Hs294t cells with anti-miR-106b resulted in the inhibition of cell proliferation as well as G1-phase arrest. They also investigated the effects of grape seed proanthocyanidins (GSPs) on the expression of miRNA-106b and its underlying molecular targets. Treatment of A375 and Hs294t cells with GSPs resulted in the suppression of miRNA-106b levels, cytotoxicity, G1-phase arrest, and the reactivation of p21/WAF1/ Cip1. Moreover, dietary GSPs significantly inhibited the growth of A375 melanoma tumor xenografts in nude mice, which was associated with reduction in the levels of miR-106b, tumor cell proliferation, and increases in the levels of p21/WAF1/Cip1 proteins. These results suggested that miRNA-106b plays a crucial role in melanoma growth and that GSPs acted as an inhibitor of miR-106b, thereby blocking melanoma growth in in vitro and in vivo models (Prasad and Katiyar, 2014). However, until now, the clinical significance of miR-106b in cutaneous melanoma has not been reported.

In the present study, qRT-PCR was performed to examine the expression of miR-106b in 15 cases of dysplastic nevi, 17 cases of melanoma metastases, and 97 cases of primary cutaneous melanoma tissue samples. We found that there were significant differences in the pattern of relative miR106b expression. High miR-106b expression was correlated with Breslow thickness, tumor ulceration, and advanced clinical stage. Thus, overexpression of miR-106b might play a critical role in melanoma 
progression. To assess whether the expression of miR-106b was a tumor prognostic biomarker, the overall survival was investigated with respect to expression levels of miR-106b in primary cutaneous melanoma. We found that the patients with high miR-106b expression showed shorter 5-year overall survival than those with low miR-106b expression. Furthermore, multivariate regression analysis showed that the status of miR-106b expression was an independent prognostic factor for overall survival.

In conclusion, this study showed that miR-106b may contribute to the progression of cutaneous melanoma and its up-regulation may be independently associated with poor prognosis of cutaneous melanoma. This suggests that miR-106b might serve as a promising biological marker for further risk stratification in the management of cutaneous melanoma.

\section{Conflicts of interest}

The authors declare no conflict of interest.

\section{REFERENCES}

Bhatti I, Lee A, Lund J and Larvin M (2009). Small RNA: a large contributor to carcinogenesis? J. Gastro. Surg. 13: 1379-1388. Carraro G, El-Hashash A, Guidolin D, Tiozzo C, et al. (2009). miR-17 family of microRNAs controls FGF10-mediated embryonic lung epithelial branching morphogenesis through MAPK14 and STAT3 regulation of E-Cadherin distribution. Dev. Biol. 333: 238-250.

Cui Q, Yu Z, Purisima EO and Wang E (2006). Principles of microRNA regulation of a human cellular signaling network. Mol. Syst. Biol. 2: 46.

Geller AC, Miller DR, Annas GD, Demierre MF et al. (2002). Melanoma incidence and mortality among US whites, 1969-1999. JAMA 288: 1719-1720.

Hudson RS, Yi M, Esposito D, Glynn SA, et al.(2013). MicroRNA-106b-25 cluster expression is associated with early disease recurrence and targets caspase-7 and focal adhesion in human prostate cancer. Oncogene. 32: 4139-47.

Kan T, Sato F, Ito T, Matsumura N, et al. (2009). The miR-106b-25 polycistron, activated by genomic amplification, functions as an oncogene by suppressing p21 and Bim. Gastroenterology 136: 1689-1700.

Karginov FV, Conaco C, Xuan Z, Schmidt BH, et al. (2007). A biochemical approach to identifying microRNA targets. Proc.. Natl. Acad. Sci. USA 104: 19291-19296.

Lee RC, Feinbaum RL and Ambros V (1993). The C. elegans heterochronic gene lin-4 encodes small RNAs with antisense complementarity to lin-14. Cell 75: 843-854.

Li Y, Tan W, Neo TW, Aung MO, et al. (2009). Role of the miR-106b-25 microRNA cluster in hepatocellular carcinoma. Cancer Sci. 100: 1234-1242.

Luo C, Tetteh PW, Merz PR, Dickes E, et al. (2013). miR-137 inhibits the invasion of melanoma cells through downregulation of multiple oncogenic target genes. J. Invest. Dermatol. 133: 768-775.

Merrill RM (2011). Risk-adjusted melanoma skin cancer incidence rates in Whites (United States). Melanoma Res. 21: 535-540.

Nana-Sinkam SP and Croce CM (2011). MicroRNAs as therapeutic targets in cancer. Transl. Res.157: 216-225.

Prasad R and Katiyar SK (2014). Down-regulation of miRNA-106b inhibits growth of melanoma cells by promoting G1-phase cell cycle arrest and reactivation of p21/WAF1/Cip1 protein. Oncotarget 5: 10636-10649.

Slaby O, Jancovicova J, Lakomy R, Svoboda M, et al. (2010). Expression of miRNA-106b in conventional renal cell carcinoma is a potential marker for prediction of early metastasis after nephrectomy. J. Exp. Clin. Cancer Res. 29: 90.

Terando A, Sabel MS and Sondak VK (2003). Melanoma: adjuvant therapy and other treatment options. Curr. Treat. Options Oncol. 4: 187-199.

Wang X, Han L, Zhang A, Wang G, et al. (2011). Adenovirus-mediated shRNAs for co-repression of miR-221 and miR-222 expression and function in glioblastoma cells. Oncol. Rep. 25: 97-105.

Wang X, Zhang H, Zhang A, Han L, et al. (2012). Upregulation of miR-20a and miR-106b is involved in the acquisition of malignancy of pediatric brainstem gliomas. Oncol. Rep. 28: 1293-1300.

Wang YX, Zhang XY, Zhang BF, Yang CQ, et al. (2010). Initial study of microRNA expression profiles of colonic cancer without lymph node metastasis. J. Dig. Dis. 11: 50-54.

Yau WL, Lam CS, Ng L, Chow AK, et al. (2013). Over-expression of miR-106b promotes cell migration and metastasis in hepatocellular carcinoma by activating epithelial-mesenchymal transition process. PloS One 8: e57882. 